Investigación Descriptiva, Correlacional o Cualitativa

Pensar en Movimiento:

Revista de Ciencias del Ejercicio y la Salud

ISSN 1659-4436

Vol. 13, No.2, pp. 1- 15

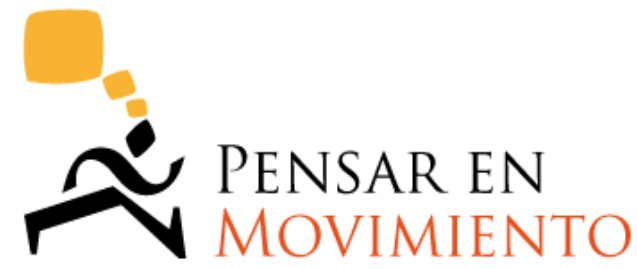

REVISTA DE CIENCIAS DEL EJERCICIO Y LA SALUd

\title{
COMPORTAMIENTO NEUROMUSCULAR POSTERIOR A LA COMPETENCIA EN JUGADORES PROFESIONALES DE FÚTBOL DE COSTA RICA: UN SEGUIMIENTO TENSIOMIOGRÁFICO
}

\section{POST-COMPETITION NEUROMUSCULAR PERFORMANCE IN PROFESSIONAL FOOTBALL PLAYERS IN COSTA RICA: TENSIOMYOGRAPHIC MONITORING}

Daniel Rojas-Valverde, Lic. ${ }^{1}(B, C, D, E)$,; Randall Gutiérrez-Vargas, M. Sc. ${ }^{1}(A, B, C, D, E)$,; Braulio Sánchez-Ureña, M.Sc. ${ }^{(D, E)}{ }^{2}{ }^{2}$; Juan Carlos Gutiérrez-Vargas, M. Sc ${ }^{3}(D, E)$, ; Immanuel CruzFuentes, M. Sc. ${ }^{3(D, E)}$,; Jorge Salas-Cabrera, Ph. $D .^{2}(D, E)$,.

${ }^{1}$ Centro de Investigación y Diagnóstico en Salud y Deporte (CIDISAD)

${ }^{2}$ Programa Ciencias del Ejercicio y la Salud (PROCESA)

${ }^{3}$ Centro de Desarrollo y Rehabilitación de Salud (CEDERSA)

${ }^{123}$ Escuela de Ciencias del Movimiento Humano y Calidad de Vida randall.gutierrez.vargas@una.cr

Facultad de Ciencias de la Salud, Universidad Nacional, Heredia, Costa Rica

Manuscrito recibido: 15/05/2015; aceptado: 10/09/2015; publicado: 15/10/2015

Doi: http://dx.doi.org/10.15517/pensarmov.v13i2.19246

\section{RESUMEN}

Rojas Valverde D.; Gutiérrez Vargas, R.; Sánchez Ureña, B.; Gutiérrez Vargas, J.C.; Cruz Fuentes, I., y Salas Cabrera, J. (2015). Investigación Descriptiva Correlacional o Cualitativa. Comportamiento neuromuscular posterior a la competencia en jugadores profesionales de fútbol de Costa Rica un seguimiento tensiomiografico. Pensar en Movimiento: Revista de Ciencias del Ejercicio y la Salud, 13(2), 1-15. Objetivo del estudio: describir el comportamiento neuromuscular posterior a la competencia en jugadores profesionales de fútbol de Costa Rica por medio de tensiomiografía. Metodología: 10 jugadores masculinos con las siguientes características: $27,78 \pm 2,87$ años, estatura $175,43 \pm 4,04 \mathrm{~cm}$, peso corporal $69,00 \pm 3,68 \mathrm{~kg}, 13,17 \pm 3,88 \%$ de grasa corporal. Se realizaron mediciones tensiomiográficas del músculo recto femoral en su estado base (LB) durante la pretemporada y 24 horas posteriores a cuatro partidos oficiales. En tales evaluaciones se comparan los valores de tiempo de contracción (Tc) y desplazamiento muscular (Dm) del hemisferio no dominante (HND) y dominante (HD). Resultados: existen diferencias significativas en Tc-HD entre LB y cuarto partido ( $F=567,62 ; p=0.037)$, en Tc-HND entre LB y cuarto partido (J4) $(F=506,90 ; p<0,001)$, primer partido (J1) y cuarto partido (J4) $(F=506,90 ; p=0,009)$, tercer $(J 3)$ y cuarto partido $(F=506,90 ; p=0,001)$ y en Dm-HD entre el segundo y cuarto partido $(F=180,65 ; p=0,022)$. Conclusiones: los valores 
tensiomiográficos (Tc y $\mathrm{Dm}$ ) son similares a los reportados por estudios previos en jugadores profesionales de fútbol. Existen diferencias significativas en al menos un valor (Tc o Dm) obtenido por medio de tensiomiografía entre la LB (Tc-HD y Tc-HND), J1 (Tc-HND), J2 (Dm-HD) y J3 (Tc-HND) comparado al J4. A medida que transcurre la temporada los valores reportados se asemejan a estudios previos del fútbol internacional y tienen un comportamiento relacionado a respuesta al entrenamiento físico.

Palabras clave: tensiomiografía; fútbol; recto femoral; neuromuscular.

\section{Abstract}

Objective: To describe the neuromuscular behavior after competition in professional soccer players of Costa Rica through tensiomyographic monitoring. Methods: 10 male players (age $27.78 \pm 2.87$ years, height $175.43 \pm 4.04 \mathrm{~cm}$, weight $69.00 \pm 3.68 \mathrm{~kg}$, body fat $13.17 \pm 3.88$ $\%$ ), healthy, training 4-5 times per week. One tensiomyographic (TMG) measurement of rectus femoris was performed in its base state (LB) in preseason and about 24 hours after each of 4 official matches, comparing the values of contraction time (Tc) and muscle displacement (Dm), of the not dominant hemisphere (HND) and the dominant one (HD). Results: There are significant differences in Tc-HD between LB and fourth match $(F=$ 567.62, $p=0.037$ ), in Tc-HND between LB and fourth match ( $F=506.90, p=0.000)$, second match and fourth match $(F=506.90, p=0.009)$, third and fourth match $(F=506.90, p=$ 0.001 ) and Dm-HD between the second and fourth match $(F=180.65, p=0.022)$. Conclusions: The TMG values are similar to previous reported soccer TMG studies. There are significant differences in at least one value (Tc or Dm) obtained by TMG between LB (TcHD and Tc-HND), J1 (Tc-HND), J2 (Dm-HD) and J3 (Tc-HND) compared to J4, getting closer, as the season goes on, to values reported for international football. This behavior corresponds to that expected in response to training.

Keywords: tensiomyography; soccer; rectus femoris; neuromuscular.

El fútbol se caracteriza por requerir de una compleja gama de funciones motoras realizadas a diferentes intensidades y espontáneamente. En gran parte, el metabolismo aeróbico es el responsable de la energía producida durante el juego. Sin embargo, el metabolismo anaeróbico es el responsable de suplir energéticamente al cuerpo en carreras de alta velocidad, aceleraciones, saltos y disparos, acciones consideradas como los principales factores que definen el rendimiento deportivo (Daneshjoo, Rahnama, Mokhtar \& Yusof, 2013; Stolen, Chamari, Castagna \& Wisloff, 2005). La repetición constante, principalmente durante los entrenamientos de estas acciones, puede inducir a la fatiga muscular. El incremento de la intensidad y frecuencia de las competiciones hace más necesaria la optimización de la recuperación posterior a la actividad debido a que el tiempo para volver a los parámetros ideales es menor (Andersson et al., 2008).

Se han registrado modificaciones en la condición muscular posterior a la competencia, lo anterior como consecuencia de la fatiga. Estos cambios son principalmente: disminución de la fuerza voluntaria e incrementos de marcadores sanguíneos de daño muscular, pero no se han registrado las respuestas contráctiles neuromusculares (Duffield et al., 2012).

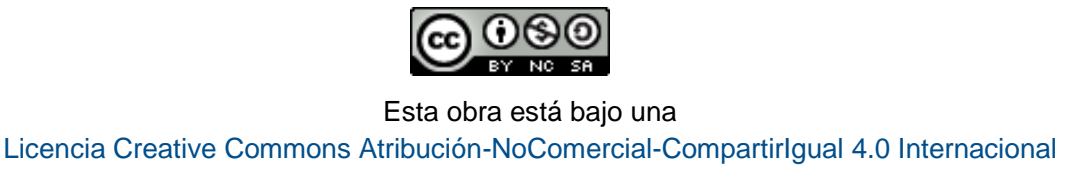


Según Gómez-Campos, Cossio-Bolaños, Brousett-Minaya y Hochmuller-Fogaca (2010) existen dos tipos de fatiga, la central (alteración de neurotransmisores) y la periférica (alteración neuromuscular o de la función metabólica). El método de Tensiomiografía (TMG) brinda información acerca de la tensión o tono muscular (Kokkonen, Nelson \& Cornwell, 1998; Rey, Lago-Peñas \& Lago-Ballesteros, 2012; Valencic \& Knez, 1997), velocidad de contracción (Simunic, Rozman \& Pisot, 2005), tipo predominante de fibra muscular esquelética (Rey, Lago-Peñas \& Lago-Ballesteros, 2012 y Valencic \& Knez, 1997), fuerza de forma indirecta proporcional al del máximo desplazamiento radial del vientre muscular (Dm) (Valencic \& Djodjevic, 2001) y fatiga (Krizaj, Simunic \& Zagar, 2008; Rey, Lago-Peñas \& Lago-Ballesteros, 2012; Valencic \& Knez, 1997). La TMG ofrece información acerca del tiempo de contracción (Tc) y el Dm durante una contracción involuntaria como respuesta a un estímulo eléctrico.

El Tc determina el tiempo que transcurre desde que finaliza el tiempo de reacción (Td) (10\% de Dm) hasta que el músculo alcanza el 90 \% de la deformación máxima (GarcíaManso, et al., 2010). Esta evaluación puede determinar el tipo de fibra predominante en el músculo, ya que un valor de Tc por debajo del promedio en situación basal se puede relacionar a un predominio de fibras tipo II, pero un valor por encima del promedio en situación basal puede indicar predominancia de presencia de fibras tipo I, especialmente si sobrepasa los $30 \mathrm{~ms}$. Asimismo, un incremento en la velocidad de contracción que puede indicar una pérdida de la fuerza muscular (Rey, Lago-Peñas \& Lago-Ballesteros, 2012; Rusu et al., 2013; Valencic \& Knez, 1997).

El Dm: "viene dado por el desplazamiento radial del vientre muscular expresado en milímetros. Representa y evalúa la rigidez muscular" (Dahmane et al., 2001; García-Manso et al., 2010; Krizai, Simunic \& Zagar, 2008; Simunic, 2003; Valencic, Knez \& Simunic, 2001). Por un lado, una disminución del $\mathrm{Dm}$ puede indicar un aumento en el tono o tensión muscular (Kokkonen, Nelson \&Cornwell, 1998; Rey, Lago-Peñas \& Lago-Ballesteros, 2012; Valencic \& Knez, 1997), así como el aumento en el Tiempo de Recuperación (Tr) puede indicar una condición de fatiga (Krizaj, Simunic \& Zagar, 2008; Rey, Lago-Peñas \& LagoBallesteros, 2012; Valencic \& Knez, 1997).

En el fútbol al usar la TMG se han evaluado las características neuromusculares en diferentes situaciones como las siguientes: efectos de exposición al frío (García-Manso et al., 2011b), intervenciones de recuperación (Rey, Lago-Peñas, Lago-Ballesteros y Casais, 2012), diferencias por posiciones de juego (Rey, Lago-Peña \& Lago-Ballesteros, 2012), evaluación de la efectividad del tratamiento del ligamento cruzado anterior (Álvarez-Díaz et al., 2014), dominancia lateral (Álvarez-Díaz et al., 2014) y diferentes tipos de entrenamientos (Rusu et al., 2013).

Con la capacidad de evaluación tecnológica no invasiva e individual de los músculos mediante la TMG, desde un punto de vista de control de fatiga neuromuscular, se obtiene información valiosa, inmediata y precisa de la condición muscular de los deportistas. Con estos datos se puede mantener un constante control y seguimiento del estado de fatiga mediante la respuesta nerviosa y de tono muscular.

Si bien, en la literatura científica existe amplio conocimiento sobre la evaluación de la fatiga, no se han estudiado parámetros neuromusculares involucrados a partir de métodos de exploración no invasiva como lo es la TMG, durante periodos competitivos en el fútbol. 
Sumado a lo anterior, no se han reportado los comportamientos de fatiga y entrenamiento de tren inferior en poblaciones de deportes de conjunto como el fútbol durante torneos oficiales.

El propósito del presente estudio fue describir el comportamiento neuromuscular posterior a la competencia en jugadores profesionales de fútbol de Costa Rica por medio de tensiomiografía.

\section{METODOLOGÍA}

\section{Sujetos.}

Los participantes del estudio fueron 10 jugadores masculinos de fútbol $(27,78 \pm 2,87$ años, estatura 175,43 $\pm 4,04 \mathrm{~cm}$, peso corporal $69,00 \pm 3,68 \mathrm{~kg}, 13,17 \pm 3,88 \%$ de grasa corporal), saludables, que entrenan $4-5$ veces por semana y compiten regularmente 1-2 veces a la semana, pertenecientes a un equipo profesional de la Primera División Costarricense de Fútbol, durante la pretemporada (Línea Base) y posterior a los primeros cinco juegos de inicio de temporada del Torneo Invierno 2014. Los mismos participaron de forma voluntaria y con el respaldo del cuerpo técnico y el club, como parte de su control de fatiga durante el torneo. Los sujetos no reportaron desórdenes neurológicos, consumo regular de drogas o afecciones musculares, no se evaluaron atletas con alguna lesión diagnosticada o aparente. Todos los juegos fueron realizados en terrenos sintéticos con medidas oficiales.

\section{Instrumentos.}

La caracterización de los participantes se realizó a partir de la medición de peso corporal con una báscula digital (sensibilidad $\pm 0,1 \mathrm{~kg}$ ) (Elite Series BC554, TanitaIronman $\AA$, Illinois, United States of America) y para conocer la talla de los futbolistas se utilizó un tallímetro de pared. La composición corporal de los jugadores se obtuvo a partir de una absorciometría dual por rayos x (DEXA) (General Electric enCORE 2011®, software versión 13.6, Wisconsin, United States of America), el cual cuantifica el porcentaje de grasa corporal. El Coeficiente de Correlación Interna es 0,6 para masa grasa (Norcross \& Van Loan, 2014).

Se utilizó un tensiomiógrafo (TMG System 100, Ljubljana, Slovenia), para determinar la condición muscular base de los sujetos y el debido seguimiento posterior en cuatro partidos de competición. En el análisis de la información se empleó el software (TMG 100 Software 3.0). Lo anterior se realizó mediante la cuantificación del tiempo de contracción (Tc) (expresada en ms) y deformación/desplazamiento del vientre muscular (Dm) (expresada en $\mathrm{mm}$ ) del músculo recto femoral (RF) tal y como lo realizó Rusu et al., (2013), García-García (2013) y García-Manso et al., (2011), quienes defienden que los músculos biarticulares son los músculos óptimos para este tipo de evaluaciones por su particularidad de ser biarticulares y por el rol que estos juegan en los deportes con estas dinámicas de movilidadestabilización continua. García-García (2013) indica que existe una relación positiva entre el $\mathrm{VO}_{2 \text { máx }}$ y Watts máx en ciclistas y el Dm, en músculos biarticulares como el RF y el bíceps femoral. 
La evaluación TMG se realiza por medio de una contracción involuntaria mediante una descarga eléctrica. La intensidad de los impulsos eléctricos según la capacidad del equipo es de entre $1 \mathrm{~mA}-110 \mathrm{~mA}$, con una duración de fase de $1 \mathrm{~ms}$ y una forma de onda monofásica cuadrangular (Rey, Lago-Peñas \& Lagos-Ballesteros, 2012). Las descargas eléctricas se realizan con un estimulador artificial (TMG-S2 d.o.o., Ljubljana, Slovenia) e inician en $20 \mathrm{~mA}$ hasta el máximo desplazamiento mecánico del vientre muscular, similar a lo indicado por Hunter, et al., (2012). Para provocar el máximo desplazamiento mecánico se realizaron incrementos graduales de $10 \mathrm{~mA}$ cada descarga (Ditroilo, Smith, Fairweather \& Hunter, 2013).

Para la transferencia eléctrica del estimulador al cuerpo se utilizan electrodos cuadrangulares $(5 \times 5 \mathrm{~cm})$ y adhesivos (TheraTrode $\AA$, TheraSigma, California, United States of America). La colocación de los electrodos fue en el punto medio de 2,5 a 3 centímetros de los bordes internos de los electrodos (Tous-Fajardo et al., 2010), el ánodo se coloca proximal y el cátodo distal (Rey, Lago-Peñas \& Lagos-Ballesteros, 2012), el sensor Dc-Dc Trans-Tek® (GK 40, Panoptik d.o.o., Ljubliana, Slovenia), se ubica en la posición perpendicular a la superficie del músculo en el punto de mayor diámetro muscular (GarcíaManso, et al., 2010), con una presión inicial de 1,5 x10-2 N/mm2 (Ditroilo, Smith, Fairweather, Hunter, 2013), según la guía anatómica para electromiografía (Delagi, Lazzetti, Perotto, \& Morrison, 2011).

La resolución del equipo según el fabricante (TMG-BMC Ltd.) es de $2 \mu \mathrm{m}$ y un error de 2 micras. La ICC para recto femoral es Tc $=0,92$ y para $\mathrm{Dm}=0,94$ (Benítez-Jiménez, Fernández-Roldán, Montero-Doblas, \& Romacho-Castro, 2013), respaldado por RodríguezMatoso et al., (2010) (Tc, $\alpha=0,97 ; \mathrm{Dm}, \alpha=0,92$ ).

El presente estudio constó de tres fases. En la primera se llevó a cabo una sesión de información y reclutamiento con los posibles participantes y encargados del cuerpo técnico. En la segunda fase se procedió a realizar dos sesiones de evaluación tensiomiográfica para obtener la condición base neuromuscular y antropométrica de los participantes. En esta sesión se evaluaron en ambos hemicuerpos (HD: Hemisferio Dominante y HND: Hemisferio No Dominante) las variables de: Tc y Dm, del músculo RF.

Posteriormente se realizó la respectiva medición antropométrica (peso, talla y porcentaje de grasa). En la tercera fase se hizo un control bilateral del músculo RF previo al inicio del campeonato llamada línea base (LB) y 24 horas posteriores a cuatro partidos oficiales del torneo nacional (partido 1 [J1], partido 2 [J2], partido 3 [J3], partido 4 [J4]), en la cual se realizaron cinco partidos oficiales en un lapso de 24 días y se tomaron mediciones de los primeros dos y últimos dos juegos.

Las mediciones base se llevaron a cabo en la sede del Centro de Investigación y Evaluación en Salud y Deporte (CIDISAD) de la Escuela Ciencias del Movimiento Humano y Calidad de Vida, Universidad Nacional, Costa Rica y se realizaron en unos cuartos controlados a una temperatura de entre 22 y $23{ }^{\circ} \mathrm{C}$ (similar a lo indicado por Rey, LagoPeñas y Lagos-Ballesteros, 2012), en dos sesiones coordinadas con el cuerpo técnico del club de fútbol; por logística de los evaluadores se distribuyeron a conveniencia en dos días consecutivos, en los cuales el jugador no recibía ningún estímulo deportivo o carga de trabajo 24 horas previas a la evaluación (Bandeira, Muniz, Abreu, Nohama, y Borba, 2012). Para determinar la línea base se promediaron las evaluaciones de las dos sesiones. Estas $-5-$ 
medidas antropométricas y tensiomiográficas fueron realizadas por el mismo evaluador. Las mediciones de control se tomaron previamente a cada uno de los entrenamientos, un día posterior a cada partido, en cuarto aislado junto al terreno de juego.

La evaluación tensiomiográfica requiere que el atleta se coloque en posición decúbito supino, sin realizar ningún movimiento, no debe haberse colocado ningún producto tópico previo a la prueba. Para que se dé una evaluación correcta, la posición debe asegurar una postura de relajación del músculo por evaluar para lo cual se debe colocar cuñas que nos permitan lograr mantener la articulación en reposo, en un ángulo de $60^{\circ}$ de flexión (Rodríguez-Matoso, et al., 2010).

Por lo tanto, es necesario seguir de manera fiel el protocolo previamente establecido para cada evaluación (García-Manso et al., 2010), ya que se logró comprobar que una diferencia en la colocación del sensor y electrodos puede dar alteraciones en los diferentes puntos evaluados por parte del equipo.

\section{Análisis Estadístico.}

Para representar cada una de las características de la muestra se implementó estadística descriptiva por medio del cálculo de valores de la media $(\mathrm{m})$ y sus respectivas desviaciones estándar ( \pm DS). La fiabilidad intraevaluador se realizó mediante pruebas de correlación de Pearson (r) (Abreu, Elizalde \& Martí, 2007; Campo-Arias \& Herazo, 2010) para los cinco momentos de evaluación, en los dos valores neuromusculares obtenidos de la TMG de ambos hemicuerpos HD y HND.

Se comprobó la normalidad de los datos de cada una de las variables mediante la prueba Shapiro-Wilk, posteriormente se prosiguió con el análisis de los datos de Tc y Dm y del músculo RF de la media de la línea base de ambos hemicuerpos comparado con la media de los valores posteriores a cada partido mediante un análisis de varianza (ANOVA) de medidas repetidas. Se utilizó el Paquete Estadístico para las Ciencias Sociales (SPSS) (IBM, SPSS Statistics, V. 21.0 Chicago, IL, USA). El nivel de significancia utilizada fue de $p<0,05$.

\section{RESULTADOS}

La correlación de Pearson entre los valores de fiabilidad intraevaluador en las evaluaciones neuromusculares arrojaron los siguientes valores: para Tc-HD $r=0,63-0,96$, $\mathrm{p}<0,05$; para Tc-HND $r=0,76-0,98, \mathrm{p}<0,05$; para DM-HD $r=0,66-0,90$, $p<0,05$ y para DM-HND $r=0,31-0,86, p<0,05$ entre los valores de LB-J1, J3LB, J3-J1, J3-J4, J4-LB y J4-J1. La tabla1 indica la comparación entre la LB, $\mathrm{J} 1, \mathrm{~J} 2$ y $\mathrm{J} 3$ con el J4, debido a que no existen diferencias significativas entre los partidos restantes entre sí o con la medición LB. 
Tabla 1.

Comparación de la media de valores neuromusculares del RF de LB, J1, J2 y J3 con el J4 posterior a cada uno de los partidos competitivos mediante ANOVA de medidas repetidas.

\begin{tabular}{|c|c|c|c|}
\hline Medición & $\begin{array}{l}\mathrm{Tc}=\mathrm{ms} \\
\mathrm{Dm}=\mathrm{mm}\end{array}$ & $M \pm D S$ & J4 (valor $p$ ) \\
\hline \multirow[t]{4}{*}{ LB } & Tc-HD & $32,09 \pm 4,75$ & $0,037^{*}$ \\
\hline & Tc-HND & $32,81 \pm 3,94$ & $0,000^{* *}$ \\
\hline & Dm-HD & $8,47 \pm 1,92$ & ns \\
\hline & Dm-HND & $8,74 \pm 1,86$ & ns \\
\hline \multirow[t]{4}{*}{ J1 } & Tc-HD & $31,39 \pm 4,58$ & ns \\
\hline & Tc-HND & $32,55 \pm 5,07$ & $0,009^{\star *}$ \\
\hline & Dm-HD & $8,64 \pm 2,63$ & ns \\
\hline & Dm-HND & $9,38 \pm 1,80$ & ns \\
\hline \multirow[t]{4}{*}{ J2 } & Tc-HD & $31,40 \pm 4,40$ & ns \\
\hline & Tc-HND & $32,17 \pm 4,85$ & ns \\
\hline & Dm-HD & $7,81 \pm 2,06$ & $0,022^{*}$ \\
\hline & Dm-HND & $8,40 \pm 2,19$ & ns \\
\hline \multirow[t]{4}{*}{ J3 } & Tc-HD & $31,16 \pm 4,49$ & ns \\
\hline & Tc-HND & $31,75 \pm 4,86$ & $0,001^{* *}$ \\
\hline & Dm-HD & $8,73 \pm 2,33$ & ns \\
\hline & Dm-HND & $9,20 \pm 1,97$ & ns \\
\hline \multirow[t]{4}{*}{ J4 } & Tc-HD & $28,73 \pm 4,28$ & NA \\
\hline & Tc-HND & $29,20 \pm 4,79$ & NA \\
\hline & Dm-HD & $9,57 \pm 2,18$ & NA \\
\hline & Dm-HND & $10,03 \pm 1,97$ & NA \\
\hline
\end{tabular}

${ }^{*} p<0,05,{ }^{* *} p<0,01$. ns $=$ no significativo. $N A=$ No aplica comparación. $D m=$ desplazamiento muscular, $T c=$ tiempo de contracción, $H D=$ hemisferio dominante, $H N D=$ hemisferio no dominante. $M=$ media, $D S=$ desviación estándar.

Fuente: elaboración propia.

La tabla 1 muestra la presencia de diferencias significativas en el comportamiento del Tc-HD entre LB y J4 ( $F=567,62, p=0,037)$. También, existen diferencias significativas en Tc-HND entre LB y J4 ( $F=506,90, p<0,001)$, J1 y J4 $(F=506,90, p<0,009)$, J3 y J4 $(F=506,90, p=0,001)$. En el caso del Dm$\mathrm{HD}$ las diferencias se encontraron entre J2 y J4 ( $F=180,65, p=0,022)$ 
Figura 1.

Comportamiento del Tc del HD y HND del RF posterior a cada partido competitivo.

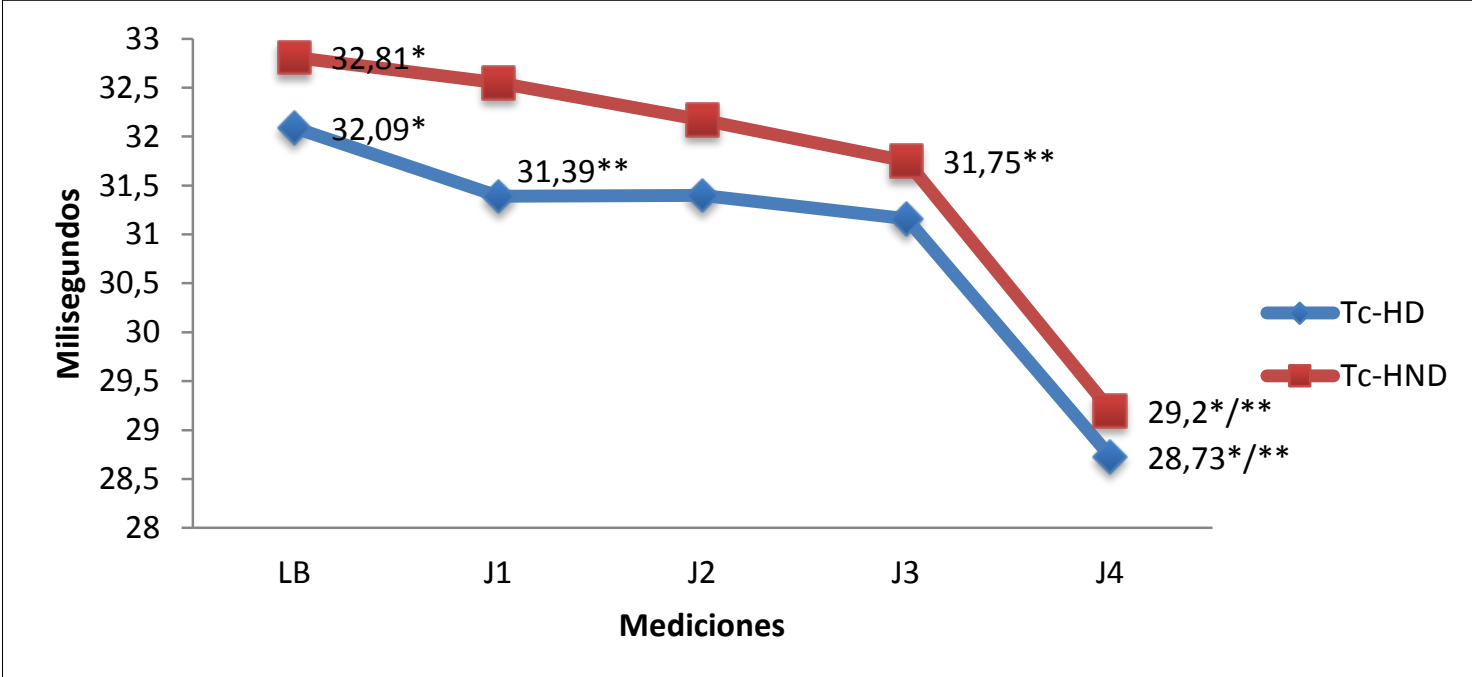

Abreviaturas: $T c=$ tiempo de contracción, $H D=$ hemisferio dominante, $H N D=$ hemisferio no

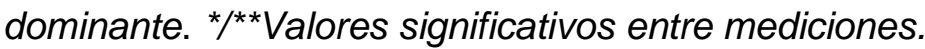

Fuente: elaboración propia.

Figura 2.

Comportamiento del Dm del HD y HND del RF posterior a cada partido competitivo.

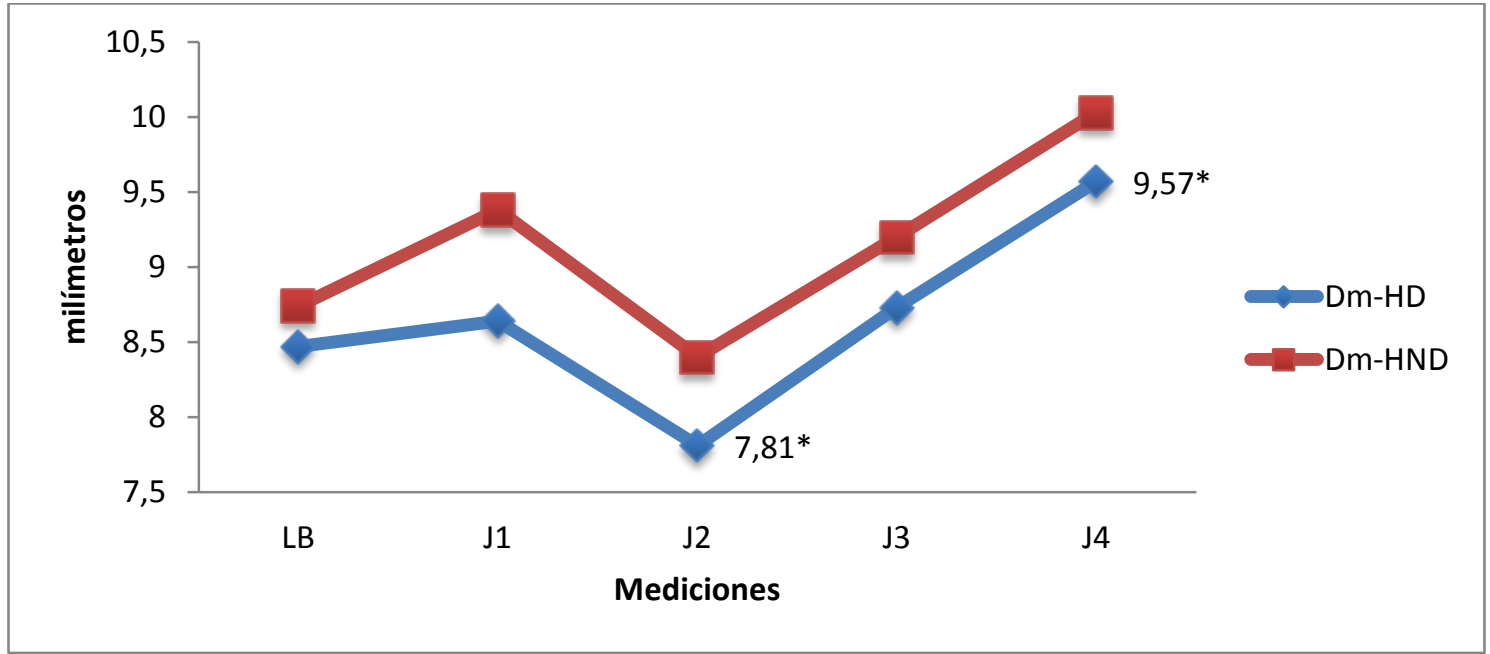

Abreviaturas: $D m=$ desplazamiento muscular, $H D=$ hemisferio dominante, $H N D=$ hemisferio no dominante. ${ }^{*}{ }^{* *}$ Valores significativos entre mediciones

Fuente: elaboración propia.

En las figuras 1 y 2 se muestran el comportamiento del Tc y del Dm del RF, en el HD y el HND, posterior a cada partido. Se puede observar el decremento gradual del Tc y el incremento gradual del DM a medida que transcurre el torneo.

$-8$

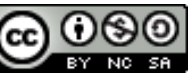

Esta obra está bajo una 


\section{DISCUSIÓN}

El presente estudio representa el primer esfuerzo por describir el comportamiento neuromuscular posterior a la competencia en jugadores profesionales de fútbol de Costa Rica por medio de tensiomiografía.

Durante la práctica del fútbol se combinan acciones intermitentes de alta intensidad y corta duración y ejercicio de base aeróbico (Daneshjoo et al., 2013; Stolen et al., 2005). Este tipo de esfuerzos explosivos tienen un impacto sobre las características morfológicas y mecánicas de los músculos del tren inferior, el aumento del tono muscular a causa de la activación aguda neural (Kubo, Kanehisa \& Fukunaga, 2001) tal y como se evidencia en el estudio de García-Manso et al., (2012), los cuales reportan el comportamiento del bíceps braquial durante el ejercicio de alto volumen y alta carga, el mismo muestra un aumento gradual del tono muscular durante e inmediatamente posterior al ejercicio, sin embargo, se genera una disminución gradual (aumento del Dm), hasta 15 minutos después de detener la actividad física, los estudios existentes en este campo no reportan lo que sucede 24 horas posterior al ejercicio físico. Sin embargo, como se evidencia en esta investigación, los futbolistas evaluados 24 horas después de la presentación deportiva, no presentan diferencias en los primero tres juegos que fueron sometidos a evaluación, es precisamente en el cuarto juego (último partido) que se denota una diferencia sobre la línea base, generando una disminución del tiempo de contracción.

La pérdida de fuerza y potencia se ha relacionado a una disminución en el tono muscular producto de la reducción que se genera en la habilidad de producir fuerza en poco tiempo durante las contracciones musculares (Kokkonen, Nelson \& Cornwell, 1998). Lo anterior se respalda por Pisot et al., (2008), quienes rescatan que los efectos del reposo posterior a 35 días en cama producen un aumento del Dm y un aumento del Tc. Asimismo, la respuesta a la fatiga muestra un comportamiento similar, inmediatamente posterior a la participación de los atletas en una ultratriatlón (García-Manso et al., 2011), lo cual refleja un aumento de los valores de Dm y Tc para bíceps femoral, pero nuevamente no se muestran valores 24 horas posteriores a la actividad.

Para explicar este comportamiento del Tc y Dm es importante considerar muchos factores como: procesos de fatiga, tipo de actividad realizada, momento de la temporada y procesos competitivos, así como el momento en que se realiza la evaluación y los espacios de descanso previo a la misma, lo anterior debido a que el aumento del Tc y un aumento del Dm no solamente se puede explicar debido a fatiga o desentrenamiento. Lo anterior es respaldado por Rusu et al., (2013), quienes indican que un aumento de Dm y el Tc es debido a un efecto normal al entrenamiento contra resistencia, con una duración de 22 días, como respuesta muscular crónica al ejercicio por la mejora en el reclutamiento muscular e hipertrofia (Saguil, 2005). Estos datos coinciden con los resultados obtenidos en esta investigación, ya que en el $\mathrm{J} 2$ hubo un aumento de desplazamiento en el hemisferio dominante (HD) de 7,81 a 9,57 milímetros con respecto al J4.

En el fútbol, los parámetros de Tc y Dm se diferencian con respecto a otros deportes como los de resistencia, por el esfuerzo anaeróbico y componentes de velocidad y potencia que el fútbol requiere, además, es importante rescatar que estudios recientes indican que existe un incremento en valores de Dm y valores más elevados de Tc al comparar los datos 
de fútbol con deportes de potencia pura (carrera, saltos) (Rey, Lago-Peñas \& LagoBallesteros, 2012). En el caso de los futbolistas costarricenses participantes de esta investigación el promedio de Tc en el J4 fue de 21,66 ms, y 9,08 para Dm. Como se puede apreciar estos difieren con los reportados por García-Manso, et al., (2011) quienes obtuvieron más de 30 ms y Dm de 8,00 milímetros. Por consiguiente, la práctica de deportes que involucra una alta intensidad permite un mejor desarrollo a nivel de fibras de contracción rápida. Por su parte Dahmane et al., (2001) establecen que en deportes de resistencia se genera mayor prevalencia de fibras de contracción lenta y con alta resistencia a la fatiga.

Similar a los datos reportados por García-García (2013) en ciclistas profesionales en pretemporada ( $\mathrm{Tc}=45,9 \pm 16,2 \mathrm{~ms}, \mathrm{Dm}=7,4 \pm 2,8 \mathrm{~mm}$ ) y lo reportado por García-García, Hernández-Mendo, Serrano-Gómez y Morales-Sánchez (2013), quienes diferencian los datos obtenidos durante una pretemporada y durante el periodo competitivo en ciclistas profesionales ( $\mathrm{Tc}=35,9 \pm 5,5 \mathrm{~ms}$ [precompetitivo] y 45,9 $\pm 16,2 \mathrm{~ms}$ [competitivo], $\mathrm{Dm}=8,6$ $\pm 3,0 \mathrm{~mm}$ [precompetitivo] y 7,4 $\pm 2,8 \mathrm{~mm}$ [competitivo]), los mismos sobrepasan el límite de 30 ms en el Tc reportado por García-Manso et al., (2011) y Dahmane et al., (2001), como deportes de base aeróbica.Los datos obtenidos de jugadores de fútbol participantes de este estudio en la respuesta de Tc y Dm del RF (ver tabla 1) son similares a los obtenidos por investigaciones previas que reportan valores referencia de futbolistas de ligas profesionales, por ejemplo, Alentorn-Geli et al., (2014) (Tc = 26,8 $\pm 5 \mathrm{~ms}$; Dm = 11,2 $\pm 20,5 \mathrm{~mm}$ ), ÁlvarezDíaz, et al., (2014b) (Tc = 26,6 $\pm 4,1 \mathrm{~ms}$ [HD] y $27 \pm 5,7 \mathrm{~ms}$ [HND] y Dm = 13,6 $\pm 28,9 \mathrm{~mm}$ [HD] y 8,8 $\pm 2,9 \mathrm{~mm}$ [HND]), Rey, Lago-Peñas, Lago-Ballesteros y Casáis (2012) (Tc = 26,7 $\pm 4,7 \mathrm{~ms} ; \mathrm{Dm}=5,5 \pm 1,7 \mathrm{~mm})$, Rey, Lago-Peñas y Lago-Ballesteros (2012) $(\mathrm{Tc}=30,86 \pm$ 2,91; $\mathrm{Dm}=11,12 \pm 3,49)$ y para jugadores jóvenes $\underline{\text { Rusu et al., (2013) }}$ (Tc = 22,94 $\pm 2,08$ $\mathrm{ms} ; \mathrm{Dm}=6,57 \pm 2,8 \mathrm{~mm})$ y Gutiérrez-Vargas et al., (2015) $(\mathrm{Tc}=27,43 \pm 3,77 \mathrm{~ms} ; \mathrm{Dm}=$ $7,37 \pm 1,24 \mathrm{~mm}$ ). Los datos referencia de estudios anteriores en cuanto a Tc y Dm del RF son ligeramente superiores o inferiores comparado con los obtenidos en el presente estudio, lo que requeriría un análisis posterior para afirmar si existe o no diferencia significativa entre sí.

En la comparación de las mediciones de los datos de Tc y Dm se concluye que no existen diferencias significativas entre la LB y J1, J2 o J3, ni entre los partidos anteriores entre sí, pero si existen diferencias significativas (ver tabla 1) entre los datos de Tc (HD y HND) de la LB comparado con el J4, así como el Tc-HND del J1 y J3 comparado con el J4, a su vez el Dm-HD del J2 comparado con el J4. Por consiguiente, basado en los anterior se debe señalar que el $\mathrm{J} 4$ tuvo una particularidad con respecto a la LB, J1, J2 y J3, esto se podría explicar basándose en lo descrito por Rusu et al., (2013), quien indica que hay una respuesta aguda al entrenamiento en un lapso de 22 días de ejercicio físico contra resistencia, similar al tiempo en que transcurrieron las mediciones del presente estudio (24 días), hubo una disminución del Tc y un aumento del Dm. Las respuestas neuromusculares mencionadas anteriormente son respuestas contrarias a las señaladas por Pisot et al., (2008) (aumento de Tc y de Dm como respuesta al reposo prolongado), García-Manso et al., (2011) (aumento de Tc y Dm como respuesta inmediata a un evento de resistencia), Gutiérrez-Vargas et al., (2015) (aumento del Tc y Dm 24 horas posterior a un maratón). 
A partir del hecho de que no existen datos tensiomiográficos en fútbol reportados en condiciones posteriores a la competición (24 horas), el presente estudio es incomparable con investigaciones previas, pero se observa a lo largo del tiempo (24 días) respuestas similares a la exposición al ejercicio físico como lo reporta Rusu et al., (2013). Lo anterior es respaldado por la tendencia de los datos de Tc y Dm a incrementarse en la medida que los sujetos se enfrentan a partidos competitivos, es decir que en la medida que avanza la temporada los sujetos presentan condiciones cada vez más aptas para la práctica deportiva (ver fig. 1 y fig. 2), en donde los datos del J4 son más similares a datos reportados por estudios previos referentes a ligas profesionales de fútbol como Alentorn-Geli et al., (2014) y Álvarez-Díaz, et al., (2014b).

\section{CONCLUSIONES}

En conclusión, los datos obtenidos en el presente estudio son similares a estudios previos realizados en el fútbol en jugadores de ligas profesionales y existen diferencias significativas en al menos un valor (Tc o Dm) obtenido por medio de tensiomiografía entre la LB (Tc- HD y Tc-HND), J1 (Tc-HND), J2 (Dm-HD) y J3 (Tc-HND) comparado al J4. Estos valores a medida que transcurre la temporada, se asemejan a datos reportados en el fútbol internacional y que se relacionan con un comportamiento esperado como respuesta al entrenamiento físico. Lo anterior señala la necesidad de estudiar el desempeño neuromuscular a través de la temporada y no solo al inicio de la misma, así como en diferentes condiciones de entrenamiento, fatiga y lapsos de descanso.

\section{REFERENCIAS}

Abreu, R., Elizalde, J. \& Martí, A. (2007). Estudio comparativo de la alteración de la conjuntiva secundaria a una vitrectomía por membrana epirretiniana vía pars plana 20G y 25G (Archivos de Sociedad Canaria Oftalmológica n. ㅇ 18). Recuperado de http://sociedadcanariadeoftalmologia.com/archivos-de-la-sociedad-canaria-deoftalmologia/archivos-sco-n-0-18-2007/

Alentorn-Geli, E., Alvarez-Díaz, P., Ramon, S., Marin, M., Steinbacher, G., Boffa... Cugat, R. (2014). Assessment of neuromuscular risk factors for anterior cruciate ligament injury through tensiomyography in male soccer players. Knee Surgery Sports Traumatology Arthroscopy, 23(9), 2508-13. doi: http://dx.doi.org/10.1007/s00167-014-3018-1

Álvarez-Diaz, P., Alentorn-Geli, E., Ramon, S., Marin, M., Steinbacher, G., Rius, M...Cugat, R. (2014). Effects of anterior cruciate ligament reconstruction on neuromuscular tensiomyographic characteristics of the lower extremity in competitive male soccer players. Knee Surgery Sports Traumatology and Arthroscophy. doi: http://dx.doi.org/10.1007/s00167-014-3165-4

Álvarez-Díaz, P., Alertorn-Geli, D., Ramon, S., Marin, M., Steinbacher, G., Rius, M., Sijas, R., Ballester, J. \& Cugat, R. (2014b). Comparison of tensiomyographic neuromuscular characteristics between muscles of the dominant and non-dominant lower extremity in male soccer players. Knee Surgery Sports Traumatology and Arthroscophy. doi: http://dx.doi.org/10.1007/s00167-014-3298-5 
Andersson, H., Raastad, T., Nilson, J., Paulsen, G., Garthe, I. \& Kadi, F. (2008). Neuromuscular fatigue and recovery in elite female soccer: effects of active recovery. Journal of the American College of Sports Medicine, 40(2), 372-380. doi: http://dx.doi.org/10.1249/mss.0b013e31815b8497

Bandeira, F., Muniz, M., Abreu, M., Nohama, P. \& Borba, E. (2012). Can thermography aid in the diagnosis of muscle injuries in soccer athletes?. Revista Brasileira do Medicina do Esporte, 18(4), 246-251. Recuperado de http://dx.doi.org/10.1590/S1517$\underline{86922012000400006}$

Benítez Jiménez, A.; Fernández Roldán, K.; Montero Doblas, J.M. y Romacho Castro, J.A. (2013) Fiabilidad de la tensiomiografía (tmg) como herramienta de valoración muscular. Revista Internacional de Medicina y Ciencias de la Actividad Física y el Deporte, 13(52), 647-656. Recuperado de http://cdeporte.rediris.es/revista/revista52/artfiabilidad398.htm

Campo-Arias, A. y Herazo, E. (2010). Cocordancia intra-e interevaluadores. Revista Colombiana de Psiquiatría, 39(2), 424-432. Recuperado de http://www.scielo.org.co/pdf/rcp/v39n2/v39n2a15.pdf.

Dahmane, R., Valencic, V., Knez, N., Erzen, I. (2001). Evaluation of the ability to make noninvasive estimation of muscle contractile properties on the basis of the muscle belly response. Medical Biologic Engineering and Computing, 39(1), 51-5. doi: http://dx.doi.org/10.1007/BF02345266

Daneshjoo, A., Rahnana, N., Mokhtar, A.H. \& Yusof, A. (2013). Bilateral and Unilateral Asymmetries of Isokinetic Strength and Flexibility in Male Young Professional Soccer Players. Journal of Human Kinetics, 36(1), 45-53. doi: http://dx.doi.org/10.2478/hukin$\underline{\text { 2013-0005 }}$

Delagi, E., Lazzetti, J., Perotto, A. \& Morrison, D. (2011). Anatomical Guide For The Electromyographer The Limbs and Trunk (Fifht Ed). Recuperado de https://books.google.co.cr/books/about/Anatomical Guide for the Electromyograph. html?id=uwos8W4HiQ8C\&redir esc $=y$

Ditroilo, M., Smith, I., Fairweather, M. \& Hunter, A. (2013). Long-term stability of tensiomyography measured under different muscle conditions. Journal of Electromyography and Kinesiology, 23(1), 558-563. http://dx.doi.org/10.1016/i.jelekin.2013.01.014.

Duffield, R., Murphy, A., Snape, A., Minett, G. \& Skein, M. (2012). Post-Match changes in neuromuscular function and the relationship to match demands in amateur rugby league matches. Journal of Science and Medicine in Sport, 15(3), 238-243. doi: http://dx.doi.org/10.1016/j.jsams.2011.10.003

García-García, O. (2013). Relación entre parámetros de tensiomiografía y potenciales indicadores del rendimiento en ciclistas profesionales. Revista Internacional de Medicina y Ciencias de la Actividad Física y el Deporte, 13(52), 771-781. Recuperado de Http://cdeporte.rediris.es/revista/revista52/artrelacion421.htm 
García-García, O., Hernández-Mendo, A., Serrano-Gómez, V. \& Morales-Sánchez, V. (2013). Aplicación de la teoría generalizabilidad a un análisis de tensiomiografía en ciclistas profesionales de ruta. Revista de Psicología del Deporte, 22(1), 53-60. Recuperado de http://www.rpd-online.com/article/viewFile/v22-n1-garcia garciahernadez mendo-serrano gomez-morales sanchez/894

García-Manso, J.M., Rodríguez-Matoso, D., Sarmiento, S., De Saa, Y., Vaamonde, D., Rodríguez-Ruiz, D. \& Sa Silva-Grigoletto, M.E. (2010). La tensiomiografía como herramienta de evaluación muscular en el deporte. Revista Anduluza de Medicina del Deporte, 3(3), 98-102. Recuperado de http://www.elsevier.es/es-revista-revistaandaluza-medicina-del-deporte-284-articulo-la-tensiomiografia-como-herramientaevaluacion-13154397

García-Manso, J., Rodríguez-Ruiz, D., Rodríguez-Matoso, D., De Saa, Y., Sarmiento, S. \& Quiroga, M. (2011). Assessment of muscle fatigue after an ultra-endurance triathlon using tensiomyography (TMG). Journal of Sports Sciences, 29(6), 619-625. doi: http://dx.doi.org/10.1080/02640414.2010.548822

García-Manso, J., Rodríguez-Matoso, D., Rodríguez-Ruiz, D., Sarmiento, S., De Saa, Y. \& Calderón, J. (2011b). Effect of cold-water immersion on skeletal muscle contractile properties in soccer players. American Journal of Physical and Medical Rehabilitation, 90(5), 356-363. Recuperado de http://journals.Iww.com/ajpmr/Abstract/2011/05000/Effect of Cold Water Immersion on Skeletal Muscle.2.aspx

García-Manso, J., Rodríguez-Matoso, D., Sarmiento, S., Saa, Y., Vaamonde, D., RodríguezRuiz, D. \& Da Silva-Grigoletto, M. (2012). Effect of high-load and high-volume resistance exercise on the tensiomyographic twitch response of biceps brachii. Journal of Electromyography and Kinesiology, 22(1), 612-619. doi: http://dx.doi.org/10.1016/j.jelekin.2012.01.005

Gómez-Campos, R.; Cossio-Bolaños, M.A.; Brousett Minaya, M. y Hochmuller-Fogaca, R.T. (2010). Mecanismos implicados en la fatiga aguda. Revista Internacional de Medicina y Ciencias de la Actividad Física y el Deporte, 10(40) pp. 537-555. Recuperado de Http://cdeporte.rediris.es/revista/revista40/artmecanismo171.htm

Gutiérrez-Vargas, R.., Rojas-Valverde, D., Salas-Cabrera, J., \& Sánchez-Ureña, B. (2015). Caracterización antropométrica y neuromuscular de deportistas de Costa Rica participantes en los Juegos Centroamericanos y del Caribe Veracruz 2014. Manuscrito en preparación.

Gutiérrez-Vargas, R.., Rojas-Valverde, D., Salas-Cabrera, J., Rodríguez-Montero, A., González-Cordero, M., Sánchez-Ureña, B., Gutiérrez-Vargas, J.C. \& Vargas-, D. (2015). Respuestas neuromusculares en corredores(as) aficionados(as), posterior a un maratón en condiciones extremas de calor/humedad. Manuscrito en preparación.

Hunter, A., Galloway, S., Smith, I., Tallent, J., Ditroilo, M., Fairweather, M. \& Howatson, G. (2012). Assessment of eccentric exercise-induced muscle damage of the elbow flexors by tensiomyography. Journal of Electromyography and Kinesiology, 22(3), 334-341. doi: http://dx.doi.org/10.1016/j.jelekin.2012.01.009 
Kokkonen, J., Nelson, A., Cornwel, I.A. (1998). Acute muscle stretching inhibits maximal strength performance. Research Quarterly for Exercise and Sport, 69(4), 411-415. Recuperado de http://dx.doi.org/10.1080/02701367.1998.10607716.

Krizaj, D., Simunic, B., Zagar, T. (2008). Short-term repeatability of parameters extracted from radial displacement of muscle belly. Journal of Electromyography and Kinesiology, 18(4), 645-651. Recuperado de http://dx.doi.org/10.1016/j.jelekin.2007.01.008.

Kubo, K., Kanehisa, H., Fukunaga, T. (2001.) Is passive stiffness in human muscles related to the elasticity of tendon structures?. Europpean Journal of Applied Physiology, 85(3), 226-32. Recuperado de http://link.springer.com/article/10.1007\%2Fs004210100463

Norcross, J. \& Van Loan, M.D. (2014). Validation of fan beam dual energy $x$ ray absorptiometry for body compositionn assessment in adults aged 18-45 years. British Journal of Sports Medicine, 38(4), 472-476. doi: http://dx.doi.org/10.1136/bjsm.2003.005413

Pisot, R., Narici, M., Simunic, B., De Boer, M., Seynnes, O., Jurdana, M., Biolo, G. \& Mekjavic, I. (2008). Whole muscule contractile parameter and thickness loss during 35-day bed rest. Europpean Journal of Applied Physiology, 104(2), 409-414. doi: http://dx.doi.org/10.1007/s00421-008-0698-6

Rey, E., Lago-Peñas, C., Lago-Ballesteros, J. (2012) Tensiomyography of selected lowerlimb muscles in professional soccer players. Journal of Electromyography and Kinesiology, 22(6), 866-872. Recuperado de http://dx.doi.org/10.1016/i.jelekin.2012.06.003.

Rey E., Lago-Peñas C., Lago-Ballesteros J., Casáis L (2012) The effect of recovery strategies on contractile properties using tensiomyography and perceived muscle soreness in professional soccer players. Journal of Strength Conditioning Research, 26(11), 3081-3088. doi: http://dx.doi.org/10.1519/JSC.0b013e3182470d33

Rodríguez-Matoso, D., Rodríuez-Ruiz, D., Quiroga, M.E., Sarmiento, S., De Saa, Y. \& García- Manso, J.M. (2010). Tensiomiografía, utilidad y metodología en la evaluación muscular. Revista Internacional de Medicina y Ciencias de la Actividad Física y el Deporte, 10(40), 620-629. Recuperado de http://cdeporte.rediris.es/revista/revista40/artcaracteristicas186.htm

Rodríguez-Matoso, D., Rodríguez-Ruiz, D., Sarmiento, S., Vaamonde, D., da SilvaGrigoletto, M. \& García- Manso, J.M. (2010). Reproducibility of muscle response measurements using tensiomyography in a range of positions. Revista Andaluza de Medicina del Deporte, 3(3), 81-86. Recuperado de http://www.elsevier.es/en-revistarevista-andaluza-medicina-del-deporte-284-articulo-reproducibility-of-muscleresponse-measurements-13154394

Rusu, L.D., Cosma, G.G., Cernaianu, S.M., Marin, M.N., Rusu, P.F., Cioc-Nescu, D.P., Neferu, F.N. (2013). Tensiomyography method used for neuromuscular assessment 
of muscle training. Journal of Neuroengeneering Rehabilitation, 10(67), 1-8. doi:http://dx.doi.org/10.1186/1743-0003-10-67

Saguil, A. (2005). Evaluation of the Patient with Muscle Weakness. American Familly Physician, 71(7), 1327-1336. Recuperado de http://www.aafp.org/afp/2005/0401/p1327.html

Simunic, B. (2003). Modelling of longitudinal and transversal skeletal muscle belly deformation (Tesis doctoral, Facultad de Ingenieria Electrica, Ljubljana, Eslovenia). Recuperada de http://www.tensiomyograph.de/download/Newton.pdf

Simunic, B., Rozman, S., Pisot, R. (2005). Detecting the velocity of the muscle contraction. III International Symposium of New Technologies in Sports, Sarajevo.

Stolen, T., Chamari, K., Castagna, C. \& Wisloff, U. (2005). Physiology of soccer: an update. Sports Medicine, 35(1), 501-36. doi: http://dx.doi.org/10.2165/00007256-200535060$\underline{00004}$

Tous-Fajardo, J., Moras, G., Rodríguez-Jiménez, S., Usach, R., Moreno, D \& Maffiuletti, N. (2010). Inter-rater reliability of muscle contractile property measurements using non-invasive tensiomyography. Journal of Electromyography and Kinesiology 20: 761-766. doi:10.1016/j.jelekin.2010.02.008

Valencic,V. \& Djodjevic, S. (2001). Influence of acute physical exercise on twitch response elicited by stimulation of skeletal muscles in man. Biomecanical Engineering, 2, 1-4. Recuperado desde http://www.tensiomyograph.de/download/Acute-E.pdf

Valencic, V., Knez, N. (1997). Measuring of skeletal muscle's dynamic properties. Artif Organs, 21(3), 240-242. doi: http://dx.doi.org/10.1111/j.1525-1594.1997.tb04658.x

Valencic, V., Knez, N. \& Simunic, B. (2001). Tenziomiography: detection of skeletal mscule response by means of radial muscle belly displacement. Biomecanical Engineering, 1, 1-10. Recuperado de http://www.tensiomyograph.de/download/Eval-E.pdf

Contribution: A- Funding, B- Study design, C- Data collection, D- Statistical analysis and interpretation of results, E- Manuscript preparation. 